Disclosure Information B. L. Cadilha: None. K. Dorman: None. D. Huynh: None. T. Lorenzini: None. M. Vänttinen: None. M. -R. Benmebarek: None. S. Stoiber: None. J. SuárezGosálvez: None. S. Endres: None. S. Kobold: None.

\section{P06.04 ENHANCING T CELL FUNCTION FOR CANCER IMMUNOTHERAPY BY MICRORNA MEDIATED KNOCKDOWN OF PRKAR1A}

A Poudel*, SM Rad, G Tan, AD McLellan. University of Otago, Dunedin, New Zealand

\subsection{6/jitc-2020-ITOC7.83}

Background Protein Kinase A (PKA) is a heterotetramer holoenzyme that consists of two regulatory and catalytic subunits. During $T$ cell activation, one of the regulatory subunits (PRKAR1A) localizes to the immune synapse, inhibiting several central proteins in the T-cell signalling cascade and leading to $\mathrm{T}$ cell inactivation. Previously, the disruption of localisation of PKA type I R1 $\alpha$ (PRKAR1A) to the immune synapse using disruptor peptides has been shown to improve chimeric antigen receptor (CAR) T cell function. ${ }^{1}{ }^{2}$ However, the effect of PRKAR1A knockdown in $\mathrm{T}$ cells (including CAR $\mathrm{T}$ cells) has not been studied yet. In this study, we have utilized microRNAs (miR); miR96/183 or miR155 to knockdown PRKAR1A and explored the advantages of PRKAR1A knockdown on $\mathrm{T}$ cell activation and function.

Materials and Methods MicroRNAs (miR); miR96/183 or miR155 were cloned from human genomic DNA into a sleeping beauty system under a doxycycline inducible promoter (TCE). Overexpression of miRNA and target knockdown was assessed at both transcript level (by real time RT-PCR) and/or protein level (by western blot) respectively while target validation was done by luciferase assay. The fate of PRKAR1A knockdown on Jurkat $\mathrm{T}$ cells activated with anti-CD3 and anti-CD28 antibodies were determined by measuring IL-2 production (ELISA) and CD69 surface expression (flow cytometry). The effect of miR96/183 or miR155 overexpression in primary $\mathrm{T}$ cells expressing HER2-CAR were also compared.

Results We efficiently overexpressed both miRNAs and downregulated PRKAR1A expression in HEK293 cells at both mRNA and protein level. Luciferase assay confirmed miRNA mediated specific knockdown of PRKAR1A; mutated 3'UTR of PRKAR1A was used as negative control. Overexpression of miRNAs also downregulated PRKAR1A expression in Jurkat cells which resulted in enhanced activation (CD69 expression) and IL-2 production following anti-CD3/CD28 stimulation compared to untransfected controls (with normal PRKAR1A expression). Additionally, miRNA 96/183 and miRNA155 were found to target inhibitory proteins of TCR signalling such as CTLA4, Foxo3 and ptpn2 and resulted in superior $\mathrm{T}$ cell function. A third-generation lentiviral system has been optimised to express either miR96/183 or miR155 and HER2CAR in the same vector and currently we are assessing the effect of PRKAR1A knockdown on primary CAR T cells.

Conclusions Overexpressing miRNA for knockdown of inhibitory proteins could be an efficient way of enhancing $\mathrm{T}$ cell function against solid tumours. Additionally, co-expressing CAR and miRNAs using lentiviral system would benefit such approaches for cancer immunotherapy.

\section{REFERENCES}

1. Newick $K, O^{\prime}$ Brien $S$, Sun J, Kappor $V$, Maceyko $S$, Lo A, Pure $E_{1}$ Moon $E_{t}$ Albelda SM. Augmentation of CAR T cell trafficking and antitumour efficacy by blocking protein kinase A (PKA) localization. Cancer Immunol Res 2016; 4(6): 541-551

2. Hussain M, Shah Z, Abbas $N$, Javeed A, Mukhtar MM, Zhang J. Targeting tumour-associated immune suppression with selective protein kinase A type (PKAI) inhibitors may enhance cancer immunotherapy. Medical Hypotheses 2016;86: 56-59

Disclosure Information A. Poudel: None. S.M. Rad: None. G. Tan: None. A.D. McLellan: None.

\section{P06.05 ID01-DELETED CAR T CELLS SHOW IMPROVED THERAPEUTIC EFFICACY IN MURINE PANCREATIC CANCER MODELS}

${ }^{1} \mathrm{AM}$ Senz ${ }^{*},{ }^{1} \mathrm{P}$ Metzger, ${ }^{1}$ RK Rubens, ${ }^{1} \mathrm{~B}$ Cadilha, ${ }^{2} \mathrm{M}$ Kirmaier, ${ }^{1} \mathrm{~S}$ Lesch, ${ }^{1} \mathrm{MR}$ Benmebarek, ${ }^{2} \mathrm{~S}$ Theurich, ${ }^{3} \mathrm{P}$ Murray, ${ }^{1} \mathrm{~S}$ Endres, ${ }^{1} \mathrm{~S}$ Kobold, ${ }^{1} \mathrm{LM}$ König, ${ }^{1} \mathrm{P}$ Duewell, 'M Schnurr. ${ }^{1}$ Division of Clinical Pharmacology, University Hospital, LMU, München, Germany; ${ }^{2}$ Gene Center, Ludwig-Maximilians-Universität (LMU), München, Germany; ${ }^{3}$ Max Planck Institute of Biochemistry, Martinsried, Germany

\subsection{6/jitc-2020-ITOC7.84}

Background Indoleamine-2,3-dioxygenase 1 (IDO1) is a cytosolic enzyme that catalyzes the rate limiting reaction in the kynurenine pathway. Dendritic cells, macrophages and several tumor entities have been described to express IDO1. In the tumor microenvironment IDO1 promotes tryptophan starvation and accumulation of kynurenines which result in $\mathrm{T}$ effector cell proliferation arrest and $\mathrm{T}$ regulatory cell induction. Additionally, IDO1 possesses two immunoreceptor tyrosinebased inhibitory motifs (ITIM) that upon phosphorylation can act as docking sites for the recruitment and activation of the tyrosine phosphatases SHP-1 and SHP-2 and ultimately to an activation of the non-canonical NF-KB pathway. Whether IDO1 is expressed in $\mathrm{T}$ cells and its potential function is unknown.

Materials and Methods Using IDO1-deleted splenocytes from CD4-Cre Ido1 $1^{\mathrm{fl} / \mathrm{fl}}$ mice and WT controls, we evaluated the induction of IDO1 in T cells, as well as the effect of IDO1 in $\mathrm{T}$ cell proliferation, differentiation and metabolism. Additionally, we compared in vitro and in vivo the cytotoxic activity of anti-epithelial cell adhesion molecule (EpCAM) chimeric antigen receptor (CAR) $\mathrm{T}$ cells using pancreatic tumor cell lines.

Results IDO1 is inducible in primary mouse $\mathrm{T}$ cells upon $\mathrm{T}$ cell activation and type $\mathrm{I}$ and type II interferon signaling. Interestingly, the use of IDO1 knockout CAR T cells prolongs survival and improves tumor control compared to WT CAR T cell treatment in subcutaneous and orthotopic pancreatic cancer models. In vitro, $\mathrm{T}$ cell proliferation, differentiation and cytotoxic function is comparable in WT and IDO1-deleted T cells. RNA sequencing, metabolic and in vivo tracking studies are currently being performed to pin down IDO1-intrinsic effects on CAR T cells.

Conclusions IDO1 is expressed in T cells upon T cell receptor and IFN stimulation and appears to negatively affect tumor control mediated by CAR T cells. Specific IDO1 deletion may improve therapeutic efficacy of CAR $\mathrm{T}$ cells in solid tumors, such as pancreatic cancer.

Disclosure Information A.M. Senz: None. P. Metzger: None. R.K. Rubens: None. B. Cadilha: None. M. Kirmaier: None. S. Lesch: None. M.R. Benmebarek: None. S. Theurich: None. P. Murray: None. S. Endres: None. S. Kobold: None. L.M. König: None. P. Duewell: None. M. Schnurr: None. 